\title{
Repository corticotropin injection versus corticosteroids for protection against renal damage in a focal segmental glomerulosclerosis rodent model
}

\author{
Kyle Hayes,', Elizabeth Warner, Chris Bollinger, Dale Wright and Richard M. Fitch
}

\begin{abstract}
Background: Focal segmental glomerulosclerosis (FSGS) causes renal fibrosis and may lead to kidney failure. FSGS and its common complication, proteinuria, are challenging to treat. Corticosteroids are ineffective in many patients with FSGS, and alternative treatments often yield suboptimal responses. Repository corticotropin injection (RCl; Acthar $\left.{ }^{\circledast} \mathrm{Gel}\right)$, a naturally sourced complex mixture of purified adrenocorticotropic hormone analogs and other pituitary peptides, may have beneficial effects on idiopathic FSGS via melanocortin receptor activation.

Methods: Two studies in a preclinical (female Sprague-Dawley rats) puromycin aminonucleoside FSGS model assessed the effect of $\mathrm{RCl}$ on renal function and morphology: an 8-week comparison of a single $\mathrm{RCl}$ dose with methylprednisolone $(N=27)$, and a 12 -week chronic $\mathrm{RCl}$ dose range study $(N=34)$. Primary outcomes were proteinuria and renal pathology improvements for measures of renal fibrosis, tubular damage, glomerular injury, and total kidney injury score. Impact of RCl treatment was also determined by assessing urinary biomarkers for renal injury, podocyte expression of podoplanin (a biomarker for injury), podocyte effacement by electron microscopy, and histological staining for fibrosis biomarkers.

Results: Compared with saline treatment, $\mathrm{RCI} 30 \mathrm{IU} / \mathrm{kg}$ significantly reduced proteinuria, with a $38 \%$ reduction in peak mean urine protein levels on day 28 in the 8 -week model, and RCl $10 \mathrm{IU} / \mathrm{kg}, 30 \mathrm{IU} / \mathrm{kg}$, and $60 \mathrm{IU} / \mathrm{kg}$ reduced peak mean urine protein in the 12 -week model by 18,47 , and $44 \%$, respectively. $\mathrm{RCl}$ also showed significant dosedependent improvements in fibrosis, interstitial inflammation, tubular injury, and glomerular changes. Total kidney injury score (calculated from histopathological evaluations) demonstrated statistically significant improvements with $\mathrm{RCl} 30 \mathrm{IU} / \mathrm{kg}$ in the 8-week study and $\mathrm{RCl} 60 \mathrm{IU} / \mathrm{kg}$ in the 12-week study. $\mathrm{RCl}$ treatment improved levels of urinary biomarkers of kidney injury (KIM-1 and OPN), expression of podoplanin, and podocyte morphology. RCI also reduced levels of desmin and fibrosis-associated collagen deposition staining. Methylprednisolone did not improve renal function or pathology in this model.

(Continued on next page)
\end{abstract}

\footnotetext{
* Correspondence: Kyle.Hayes@mnk.com

Mallinckrodt Pharmaceuticals, 675 James S. McDonnell Blvd, 20-1-W,

Hazelwood, MO, USA

(c) The Author(s). 2020, corrected publication 2020. Open Access This article is licensed under a Creative Commons Attribution 4.0 International License, which permits use, sharing, adaptation, distribution and reproduction in any medium or format, as long as you give appropriate credit to the original author(s) and the source, provide a link to the Creative Commons licence, and indicate if changes were made. The images or other third party material in this article are included in the article's Creative Commons licence, unless indicated otherwise in a credit line to the material. If material is not included in the article's Creative Commons licence and your intended use is not permitted by statutory regulation or exceeds the permitted use, you will need to obtain permission directly from the copyright holder. To view a copy of this licence, visit http://creativecommons.org/ licenses/by/4.0/. The Creative Commons Public Domain Dedication waiver (http://creativecommons.org/publicdomain/zero/1. 0/) applies to the data made available in this article, unless otherwise stated in a credit line to the data.
} 
(Continued from previous page)

Conclusions: These results provide evidence supporting the improvement of FSGS with RCl, which was superior to corticosteroid treatment in this experimental model. To the authors' knowledge, this is the first evidence that a drug for the treatment of FSGS supports podocyte recovery after repeated injury.

Keywords: Focal segmental glomerulosclerosis, Repository corticotropin injection, Nephrotic syndrome, Adrenocorticotropic hormone, Melanocortin receptor, Puromycin aminonucleoside

\section{Background}

\section{Focal segmental glomerulosclerosis}

Focal segmental glomerulosclerosis (FSGS) is a disease that attacks kidney glomeruli and causes scarring, leading to kidney damage. FSGS is a renal condition frequently associated with nephrotic syndrome (NS) and is one of the most common forms of glomerular disease [1, 2]. FSGS has been observed in up to $35 \%$ of biopsies obtained from adult patients with idiopathic NS, and recent epidemiological data suggest the prevalence of FSGS is increasing $[3,4]$. Histologically, FSGS is characterized by focal lesions of the glomeruli due to podocyte damage, which correlates with loss of function in glomerular permeability, proteinuria, and cell death [1-3]. FSGS and its most common complication, proteinuria, can be difficult to treat. FSGS shows the largest decline in estimated glomerular filtration rate and highest rate of progression to end-stage renal disease among glomerulopathies [5].

\section{Current FSGS treatment}

First-line treatment for idiopathic FSGS is oral corticosteroids [6]. The mechanism of these drugs is unclear, but they are believed to act through potent immunosuppression and decreased nuclear factor-kappa B (NF$\mathrm{\kappa B})$-induced cytokine production [7]. Unfortunately, many patients develop steroid-resistant or steroiddependent FSGS. Responses to steroids are lower in adult patients with FSGS versus other NSs, with a relapse rate of $47 \%$ in initial responders and 40-60\% showing no improvement after 4 months [8]. The alternatives for patients with steroid-resistant FSGS include stronger immunosuppressants, such as calcineurin inhibitors, alkylating agents, and mycophenolate mofetil. However, these are associated with adverse effects and variable efficacy, which highlights the need for additional safe and effective options [6, 8]. Recently, an expert panel of nephrologists provided guidance on treatment of patients with NS [9]. The authors emphasized selecting therapies on the basis of disease pathophysiology and highlighted promising treatments not included in the current Kidney Disease Improving Global Outcomes guidelines, such as repository corticotropin injection (RCI; Acthar ${ }^{\circledast}$ Gel, Mallinckrodt Pharmaceuticals, Bedminster, NJ) [10].

\section{Repository corticotropin injection}

RCI contains a naturally sourced complex mixture of purified adrenocorticotropic hormone analogues and other pituitary peptides [11]. RCI is indicated to induce remission of proteinuria in either NS without uremia of the idiopathic type or NS due to lupus erythematosus [11]. An increasing body of evidence has demonstrated the efficacy of RCI in difficult-to-treat patients with steroid-resistant or steroid-dependent glomerulopathies who failed standard first-line treatments. Tumlin et al. (2017) showed a significant overall reduction in proteinuria $(p<0.001)$, with $62 \%$ partial response and $8 \%$ complete response at 6 months with RCI in 13 FSGS patients who had failed multiple first-line therapies [12]. Similar results were seen in 9 patients with treatmentresistant idiopathic membranous nephropathy (iMN), with decreased proteinuria $(p=0.013)$ and a $44 \%$ partial response, which improved further with the addition of tacrolimus [12]. This supports previous findings wherein 9 of 11 patients with iMN and 1 of 1 patient with FSGS who failed an average of 2-3 immunosuppressive therapies achieved partial or complete remission (55 and $27 \%$, respectively) $[13,14]$.

RCI activates all 5 melanocortin receptors (MCRs), each with a unique function. MC2R is the main target of activation in the hypothalamus-pituitary-adrenal axis leading to adrenal steroidogenesis and release $[15,16]$. Autonomic and neuroendocrine functions appear to be heavily regulated by MC4R [17], whereas MC1R, MC3R, and MC5R are involved in steroid-independent immunomodulation of immune cells [18]. Increasing evidence suggests that MC1R activation alone may serve as a podocyte-specific target in kidney injury [19-22].

The efficacy of MCR agonists was originally thought to be due to their steroidogenic activity through MC2R. However, Lal and colleagues [23] showed an almost 37 times greater total steroid exposure with methylprednisolone (MP) treatment compared with RCI treatment, with clinically relevant doses for multiple sclerosis (MS). In light of this finding and owing to the functional differences between endogenous cortisol activation and exogenous corticosteroids, the mechanism of action of RCI extends beyond steroidogenesis. Clinical and experimental evidence continues to suggest a potential use for RCI in treating NS and idiopathic FSGS, particularly in 
patients with disease resistant to steroids and other immunosuppressants [13, 24-26]. Previous studies have demonstrated that RCI inhibits NF- $\mathrm{kB}$ activity and suppresses proinflammatory cytokine production from immune cells, which suggests that RCI might be superior to corticosteroids for the treatment of renal fibrosis [27]. However, evidence from both preclinical and clinical studies is limited, and the exact mechanisms through which RCI demonstrates efficacy in patients with steroid resistance are still unclear.

\section{Central hypothesis}

Our hypothesis was that RCI, as a pan-MCR receptor agonist, would be superior to corticosteroids on podocyte function, renal fibrosis, and proteinuria in an experimental animal model containing podocyte damage and FSGS-type lesions characteristic of NS.

\section{Methods}

\section{Preclinical PAN model}

The current study used a modified rat puromycin aminonucleoside (PAN) model, resulting in FSGS-type lesions and podocyte damage observed in NS [1, 28, 29]. Disease induction for rodent FSGS has been described previously, with modification to dosage of PAN and route of delivery. We selected intravenous (IV) PAN delivery at $50 \mathrm{mg} / \mathrm{kg}$ with booster doses of $20 \mathrm{mg} / \mathrm{kg}$ to induce FSGS instead of one-time dosing that results in a minimal change disease phenotype $[28,30]$. Two studies were conducted with female Sprague-Dawley rats (Envigo RMS, LLC, Indianapolis, Indiana, USA; age: 6-8 weeks; weight: $166-232 \mathrm{~g})$. Due to the schedule of ongoing studies, the rats used in the 12-week study were slightly younger than those in the 8-week study. All animals were delivered with documentation confirming that they were treatment-naive and free of all tested pathogens. The first study used an 8-week PAN model, which primarily compared RCI with MP ( $N=27 ; n=3$ naive, $n=8$ each for saline [diseased], RCI $30 \mathrm{IU} / \mathrm{kg}$, and MP $2 \mathrm{mg} / \mathrm{kg}$ ). The second study used a long-term 12-week PAN model $(N=$ 34; $n=4$ naive, $n=6$ for RCI $30 \mathrm{IU} / \mathrm{kg}, n=8$ each for saline, RCI $10 \mathrm{IU} / \mathrm{kg}$, and $60 \mathrm{IU} / \mathrm{kg}$ ) with an extended dose range of RCI for the treatment of FSGS. Disease induction with PAN occurred on day $0(50 \mathrm{mg} / \mathrm{kg})$, with treatment starting on day 7 . Reinjury occurred with lower-dose PAN $(20 \mathrm{mg} / \mathrm{kg})$ on days 14,21 , and 28 in the 8 -week model, and additional injury occurred on day 35 in the 12-week model (Fig. 1). Naive controls did not receive PAN induction. Animals were sacrificed in a controlled delivery system using isoflurane followed by exsanguination.

During the 8- and 12-week studies, multiple disease endpoints were analyzed to assess renal function and kidney morphology. Primary dependent measures were levels of proteinuria and scores for renal fibrosis, tubular damage, and glomerular injury. A score for interstitial inflammation was also measured, and the 4 scores were summed to yield a total kidney injury score. Scoring was performed by a certified pathologist and was graded from 0 (normal) to 5 (severe).

\section{Experimental conditions and locations}

All experiments were performed between $8 \mathrm{AM}$ and $5 \mathrm{PM}$ central standard time. Rats were housed 3 or 4 animals per cage in standard shoebox cages (16x8x8 inches). Aspen shavings were used for bedding in home cages. All study animals were kept on a diurnal 12-h light/dark cycle, with ample light used for routine inspection and cleaning. Ambient temperatures were maintained between $65^{\circ} \mathrm{F}$ and $85^{\circ} \mathrm{F}$. Standard rat chow pellets were given freely. All animals had ad libitum access to potable water

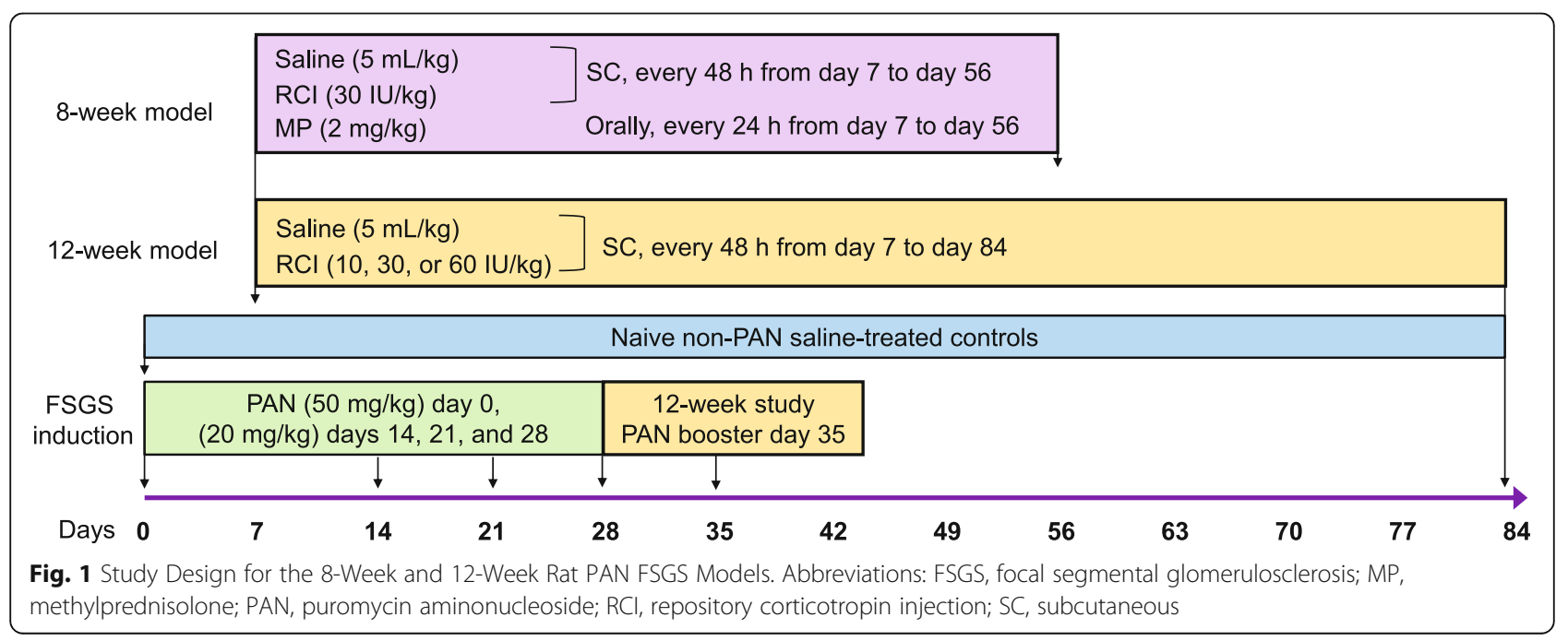


via water bottle. Rats were provided with enrichment devices such as Nylabone ${ }^{\circ}$ products, paper twists, or other devices approved by the attending vet.

All in-life experiments were conducted in the site vivarium, with dosing and urine collection completed in the controlled procedure room. Animals were observed daily, including weekends and holidays, to assess general health and well-being and were weighed at least twice per week.

All rat urine assays were performed with 24-h urine collections in metabolism cages with 1 rat per cage. Animals were normalized across $24 \mathrm{~h}$ in the metabolism cages, urine tubes were replaced, and animals remained in the metabolism cages for an additional $24 \mathrm{~h}$ for the duration of the urine collection. Animals had unlimited access to food and water. All histology procedures, including immunohistochemistry (IHC) staining, were completed on site. Enzyme-linked immunosorbent assay (ELISA) was conducted in the laboratory space adjoining the Meso Scale Discovery platform. All image analyses were completed in a designated imaging room on-site. All experiments were completed in the same building, on the same floor, and in the same wing of the floor.

\section{Sample size determination}

Animal sample size was determined using the resource equation method, with consideration of minimizing unnecessary animal usage, per our site guidelines [31]. This equation uses the following formula: $\mathrm{E}$ (degree of freedom of analysis of variance) $=$ TA (total animals) - TG (total number of groups). For the 8-week study, 27 (TA) $-4(\mathrm{TG})=23$. For the 12-week study, $34(\mathrm{TA})-5$ $(\mathrm{TG})=29$. Values greater than 20 are considered good. As naive samples were only included to confirm baseline non-disease parameters, limited numbers were used in accordance with ethical guidance to limit unnecessary animal usage.

\section{Study drugs and treatment regimens}

Dosing methodology was selected on the basis of normal treatment routes of administration. For example, in clinical settings, RCI is given subcutaneously. The most common route of administration of corticosteroids is oral dosing. The doses for RCI were determined by multiple previous in-house studies across animal models that have shown efficacy. As for MP dosing, high-dose treatment in human FSGS for prednisone is $1 \mathrm{mg} / \mathrm{kg} /$ day, with lower doses of $0.5 \mathrm{mg} / \mathrm{kg} /$ day used for maintenance [32]. Rat dosing translates to approximately 3 to $6 \mathrm{mg} / \mathrm{kg} /$ day of prednisone, or $\sim 2$ to $5 \mathrm{mg} / \mathrm{kg} /$ day of MP. We therefore selected $2 \mathrm{mg} / \mathrm{kg} /$ day of MP to avoid side effects of high-dose steroid treatment and to use a dose that is in line with other animal models of kidney injury.
RCI (10, 30, and $60 \mathrm{IU} / \mathrm{kg}$ ) and saline were administered subcutaneously, whereas MP was administered by oral gavage. In the 8-week model, FSGS was induced with 4 IV doses of PAN (days 0, 14, 21, and 28). RCI $(30 \mathrm{IU} / \mathrm{kg})$ was administered every other day beginning on day 7, whereas MP $2 \mathrm{mg} / \mathrm{kg}$ was given daily beginning on day 7. In the 12-week model, FSGS was induced with 5 IV doses of PAN (days 0, 14, 21, 28, and 35), and RCI $(10,30$, and $60 \mathrm{IU} / \mathrm{kg}$ ) was administered every other day beginning on day 7 . Naive rats were treated with saline and no PAN to mimic handling of experimental rats (Fig. 1).

All animals were treated in the morning. On days when both RCI and MP treatments were given, animals were treated by group at the same time. Animals were assessed daily by visual inspection.

\section{Urine protein collection and creatinine assays}

Urine was collected biweekly for the 8-week PAN study and weekly for the 12-week PAN study. On urine collection days, urine was initially discarded while animals acclimated to metabolic cages for $24 \mathrm{~h}$. Over the next $24 \mathrm{~h}$, urine was collected, centrifuged, aliquoted, and stored at $-80^{\circ} \mathrm{C}$ until processing. Total urine protein was measured using the pyrogallol red-molybdate dye-binding method (BioAssay Systems, Hayward, CA, Cat. \#QTPR001), and 24-h total protein was calculated to assess proteinuria. Urine creatinine $(\mathrm{uCr})$ was determined with the Jaffe method, a colorimetric plate-based assay (BioAssay Systems, Hayward, CA, Cat. \#DICT015). Two technical replicates were included for each sample, with the average of the replicates used for the final value.

\section{Meso Scale multiplex ELISA}

Urinalysis of kidney injury biomarkers osteopontin (OPN) and kidney injury molecule-1 (KIM-1) was performed via multiplex ELISA on the Meso Scale Diagnostics (Rockville, MD) Discovery system. KIM-1 and OPN were evaluated on day 28 after the third PAN booster dose $(20 \mathrm{mg} / \mathrm{kg})$, at peak proteinuria in the 8 -week model, and again at day 56 after 4 weeks of treatment following the final PAN injection. An additional time point at day 42 was added for the 12-week model, corresponding to peak proteinuria in the study. Samples were tested via rat Kidney Injury Panel-1 (Cat. \#K15162C, Meso Scale Diagnostics, Rockville, MD), and values were expressed as $\mathrm{ng} / \mathrm{mL}$. The calculated values from the ELISA were normalized for each animal to the $\mathrm{uCr}$ concentrations in $\mathrm{mg} / \mathrm{mL}$, resulting in a ratio of ng:mg of target:creatinine concentration. Two technical replicates were included for each sample, with the average of the replicates used for the final value. Because the efficacy comparison was only in the PAN treatment (diseased) groups, naive (non-diseased; non-treated) samples were 
pooled between the 8- and 12-week studies for ELISA comparisons to gain a more robust negative control baseline. Little variation in naive samples was expected based on previous experience of standard values for this age range, and animal numbers were limited in each study for ethical reasons.

\section{Histology, pathology, and IHC procedures}

Animals were sacrificed, and kidneys were immediately removed. Cross sections of the central portion of the left and right kidneys were fixed in 10\% neutral buffered formalin and processed to paraffin blocks for routine histology and IHC. In the 12-week PAN study, additional portions of renal cortex from both the left and right kidneys were fixed for analysis by electron microscopy (EM). All kidneys were stained with haematoxylin and eosin (H\&E) and periodic acid-Schiff (PAS) for pathology scoring; Masson's trichrome, Sirius Red/FastGreen, and IHC staining were used to assess fibrosis and podocyte biomarkers. Slides stained with H\&E, PAS, and Masson's trichrome were sent to Seventh Wave Laboratories (Maryland Heights, MO) for a renal pathology assessment by a board-certified veterinary pathologist who was not blinded to treatment groups, but did not have details on the specific study drug. EM samples for the 12-week study were sent to Charles River Laboratories (Durham, NC), where they were evaluated for treatment-related changes to the glomerulus by a boardcertified veterinary pathologist who was not blinded to treatment groups. All IHC staining (eg, for podoplanin [PDPN]) was performed on the Ventana (Oro Valley, AZ) Discovery Ultra automated stainer. The antibody used for PDPN was Novus (Centennial, CO) NB110962423.

\section{Quantitative image analysis}

Slides were scanned on a NanoZoomer S210 (Hamamatsu Photonics, Hamamatsu, Japan) at 20x or 40x magnification. Tissue images were analyzed through Visiopharm (Hørsholm, Denmark) quantitative image analysis software. For quantification of fibrosis, the kidney cortex was outlined at the region of interest (ROI), and interstitial fibrosis was quantitated per area of total tissue. Glomeruli from all areas of the kidney cortex were selected as ROIs from both the left and right kidney sections. An average of $\sim 200$ glomeruli per animal was selected to decrease bias due to focal pathology. Two individuals randomly identified the ROIs used in the final analyses. The software was trained to identify and quantitate staining of interest. All slides were batch processed using the same algorithm for all slides in each analysis. Naive samples were pooled for imaging analysis across studies, similar to the ELISA analysis.

\section{Statistical analysis}

Comparisons between groups were performed through 1-way or 2-way analysis of variance (ANOVA) and to check the assumption of equal variances, with appropriate post hoc tests for multiple comparisons or $t$ tests as indicated for continuous normally distributed data. Nonnormally distributed continuous and categorical data were compared with nonparametric Kruskal-Wallis (ANOVA) with post hoc tests, Mann-Whitney for 2-group comparison, or Fisher's exact test. Graphs are presented as mean \pm standard error of the mean unless otherwise noted. A significance level of $p<0.05$ was established for all statistical tests.

\section{Protocol deviations}

In the 8-week study, one naive control died after routine bleeding, which was categorized as a laboratory handling error. In the 12-week study, 2 animals in the RCI $30 \mathrm{IU} /$ $\mathrm{kg}$ group lost more than $20 \%$ of their body weight by day 47 or day 49 and were sacrificed according to our humane animal use protocol. Both animals had elevated proteinuria from disease induction, even prior to treatment, and maintained elevated proteinuria levels compared with all groups until their death. The lack of any animal deaths in the higher dose group of $60 \mathrm{IU} / \mathrm{kg}$ suggests the deaths were not likely treatment related but were caused by an extreme sensitivity to PAN injury. These animals were not included in any analyses. No additional controls or modifications were needed in this study, as the animal use guidelines implemented here account for changes in animal health, and the number of unexpected deaths was low (3/64 animals).

\section{Ethical considerations}

All study procedures, including for animal use welfare, were performed in accordance with the National Institutes of Health's Guide for the Care and Use of Laboratory Animals. The study protocol was reviewed and approved by the Institutional Animal Care and Use Committee of Mallinckrodt Pharmaceuticals.

\section{Results}

\section{Baseline characteristics}

Animals were randomized by urine protein levels to ensure no significant differences were observed between groups. Baseline body weights and urine protein were measured before disease induction (Table 1).

\section{$\mathrm{RCl}$ effect on renal function in PAN-FSGS models}

Naive (non-diseased) rats were assessed for comparison throughout our studies to confirm the disease phenotype induced by PAN. Treatment of rats with puromycin at $50 \mathrm{mg} / \mathrm{kg}$ at day 0 , followed by booster doses of $20 \mathrm{mg} / \mathrm{kg}$ at days 14, 21, and 28 in the 8-week model and an 


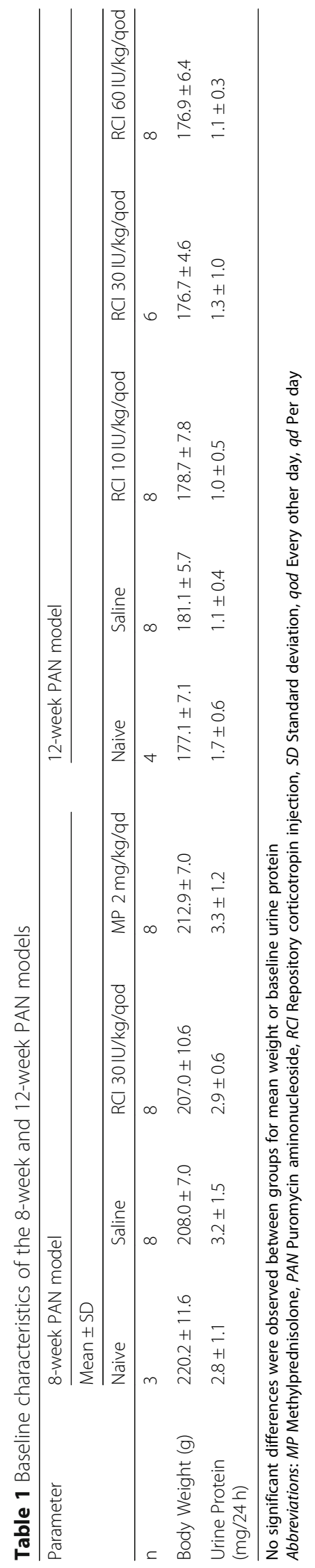




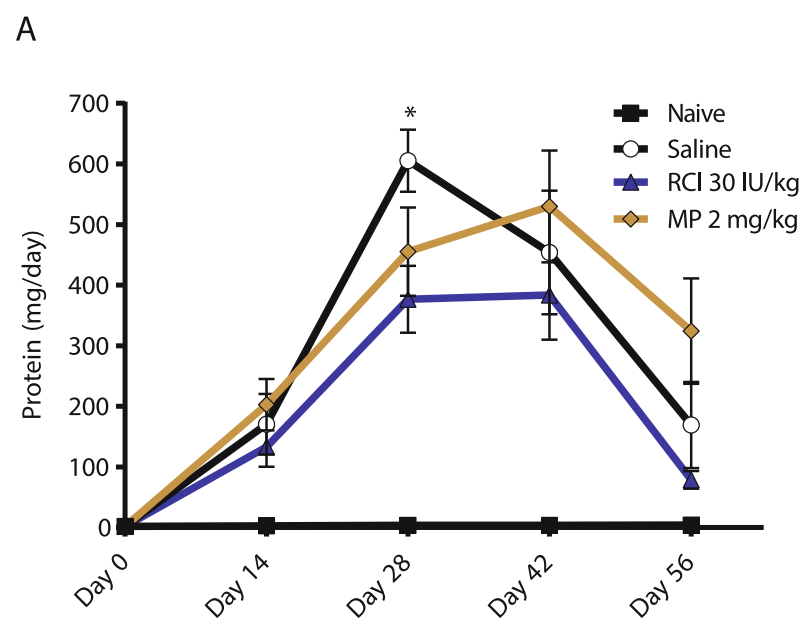

B

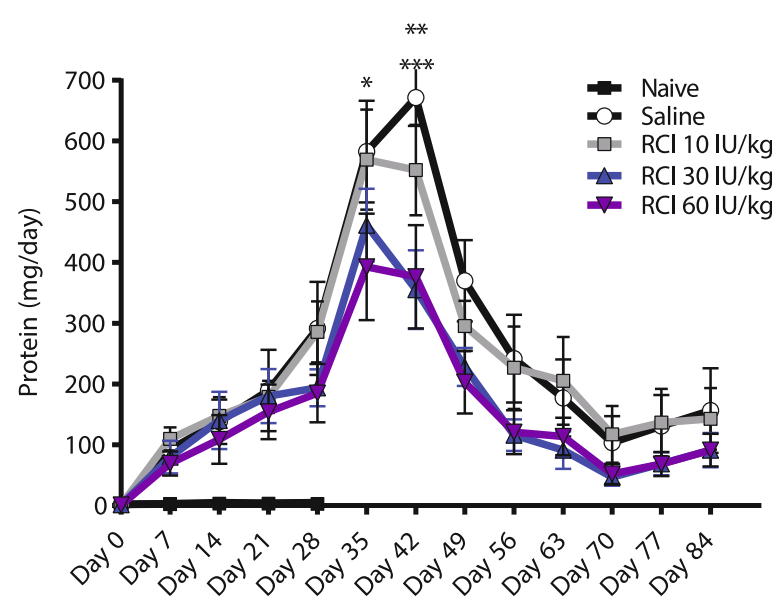

Fig. 2 Proteinuria in the 8-Week and 12-Week PAN Models. a Protein concentration (mg/24 h) in urine over the 8-week PAN study $\left({ }^{*} p<0.05\right.$ day 28 for RCl $30 \mathrm{IU} / \mathrm{kg}$ versus saline; 2-way ANOVA, Bonferroni's post hoc comparison). b Protein concentration (mg/24 h) in urine over the 12-week study ( ${ }^{*} p<0.05$ day 35 for $60 \mathrm{IU} / \mathrm{kg} \mathrm{RCl}$; ${ }^{* *} p<0.001$ day 42 for $30 \mathrm{IU} / \mathrm{kg} \mathrm{RCl}$; ${ }^{* *} p<0.0001$ day 42 for $\mathrm{RCl} 60 \mathrm{IU} / \mathrm{kg}$; all versus saline; 2-way ANOVA, Bonferroni's post hoc comparisons). In both panels, values are mean \pm standard error of the mean. Abbreviations: ANOVA, analysis of variance; MP, methylprednisolone; PAN, puromycin aminonucleoside; $\mathrm{RCl}$, repository corticotropin injection

additional dose at day 35 in the 12-week model, resulted in maximum urine protein levels of approximately $600-$ $700 \mathrm{mg} / 24 \mathrm{~h}$. RCI treatment of $30 \mathrm{IU} / \mathrm{kg}$ significantly reduced proteinuria $(p<0.05)$, with a $38 \%$ reduction in peak mean urine protein levels at day 28 compared with saline and a $29 \%$ total reduction in area under the curve (AUC) over the study period $(12,843 \mathrm{mg} / 24 \mathrm{~h}$ *d vs $18,107 \mathrm{mg} / 24$ $\mathrm{h} * \mathrm{~d}$ ) in the 8-week model (Fig. 2a). We also observed an initial 25\% decrease in peak urine protein levels with MP treatment of $2 \mathrm{mg} / \mathrm{kg} / \mathrm{d}$ at day 28 , but this reduction was lost by day 42 , and levels were nearly 2 -fold greater than with saline at day 56, with similar total AUC as saline over the study duration $(18,486 \mathrm{mg} / 24 \mathrm{~h}$ *d MP treatment vs 18 , $107 \mathrm{mg} / 24 \mathrm{~h}$ *d saline treatment). MP treatment showed nearly 4-fold higher urine protein levels than with RCI at day 56. In the 12-week RCI multidose model, peak urine protein at day 42 was reduced $18 \%$ versus saline at the lowest dose tested of $10 \mathrm{IU} / \mathrm{kg}$, with significant reductions of 47 and $44 \%$ from peak levels at the $30 \mathrm{IU} / \mathrm{kg}$ and $60 \mathrm{IU} /$ $\mathrm{kg}$ dose levels, respectively ( $p<0.001$ for each, Fig. $2 \mathrm{~b}$ ). Proteinuria AUC for the 12-week study was reduced by 35 and $39 \%$ in the $30 \mathrm{IU} / \mathrm{kg}$ and $60 \mathrm{IU} / \mathrm{kg}$ groups, respectively, compared with saline $\left(13,769 \mathrm{mg} / 24 \mathrm{~h}^{*} \mathrm{~d}\right.$ and 12,987 $\mathrm{mg} / 24 \mathrm{~h} * \mathrm{~d}$ versus $21,139 \mathrm{mg} / 24 \mathrm{~h}^{*} \mathrm{~d}$ ).

\section{Effects of $\mathrm{RCl}$ on kidney injury in PAN-FSGS models}

In PAN-treated rats, mild to moderate increases in renal fibrosis, tubular injury, and glomerular changes were observed by histopathologic analysis, with a minimal to mild increase in interstitial inflammation in both the 8- week and 12-week models. RCI treatment at 30 or 60 $\mathrm{IU} / \mathrm{kg}$ reduced these pathologic conditions, with dosedependent decreases in the 12-week study (Table 2). The calculated total kidney injury score was significantly lower with RCI $30 \mathrm{IU} / \mathrm{kg}$ in the 8-week model versus saline, mean \pm standard deviation of $5.9 \pm 1.4$ vs $8.8 \pm 2.1$ $(p<0.05)$, and with RCI $60 \mathrm{IU} / \mathrm{kg}$ in the 12 -week model versus saline, $5.1 \pm 2.5$ vs $8.8 \pm 2.4(p<0.05)$. Treatment with MP did not reduce disease histopathology or the calculated total kidney injury score compared with saline in the 8-week model (Table 2).

RCI treatment also lowered urine biomarkers of kidney injury. Concentrations of KIM-1 and OPN normalized to $\mathrm{uCr}$ (KIM-1/uCr, ng/mg) were analyzed in both 8- and 12-week models (Figs. 3 and 4). Naive samples showed little variability for KIM-1 and OPN analyses (Figs. 3 and 4). KIM-1/uCr was significantly elevated after PAN treatment plus saline at all time points in both models compared with naive controls $(p<0.01)$ (Figs. 3a, $\mathrm{b}$ and $4 \mathrm{a}-\mathrm{c})$. Rats treated with $\mathrm{RCI}$ at $30 \mathrm{IU} / \mathrm{kg}$ had significantly lower KIM-1 concentrations compared with saline on days 28 and 56 in the 8-week study $(p<0.05)$, whereas MP treatment showed higher KIM-1 levels compared with saline on day 28 and 56 on average (Fig. 3a, b). Treatment with RCI doses of 30 and $60 \mathrm{IU} / \mathrm{kg}$ significantly reduced KIM-1 concentration compared with saline on day 56 in the 12-week model $(p<0.01)$ (Fig. 4c).

The kidney injury biomarker OPN was not significantly elevated after PAN treatment plus saline at day 28 or 56 in either model (Figs. 3c, d and $4 \mathrm{~d}, \mathrm{f}$ ), but it was 


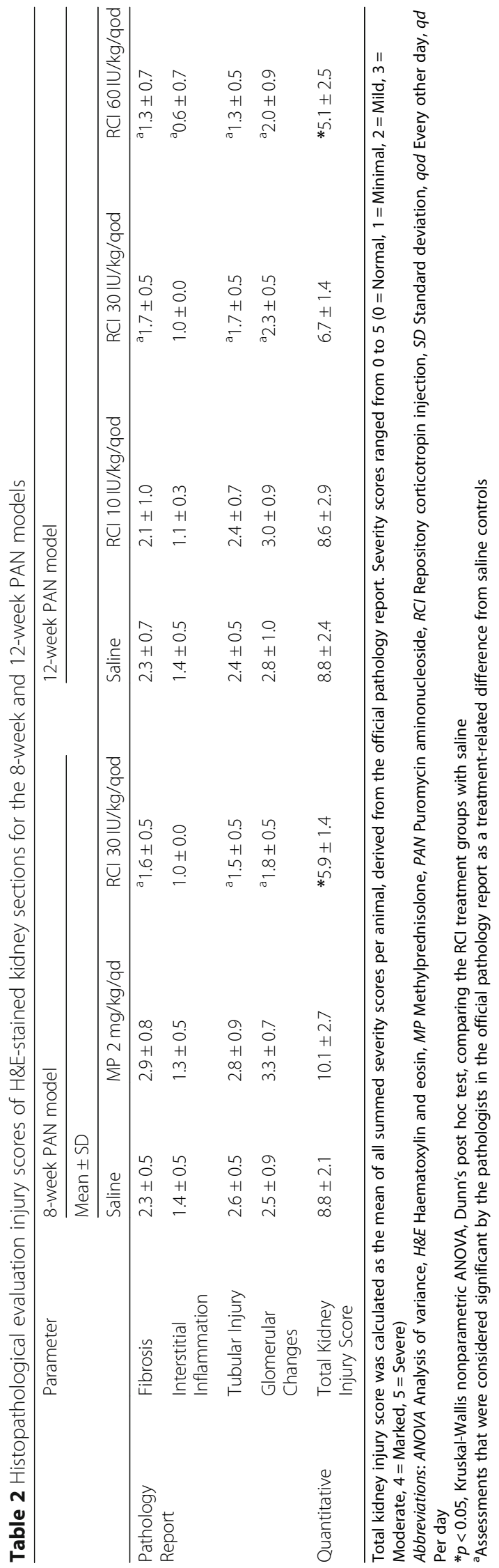




$$
\begin{array}{ll}
\text { A } & \text { KIM-1 } \\
\text { Day } 28
\end{array}
$$

Day 28

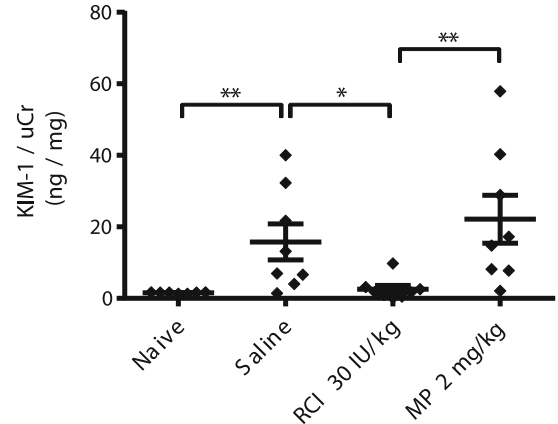

C

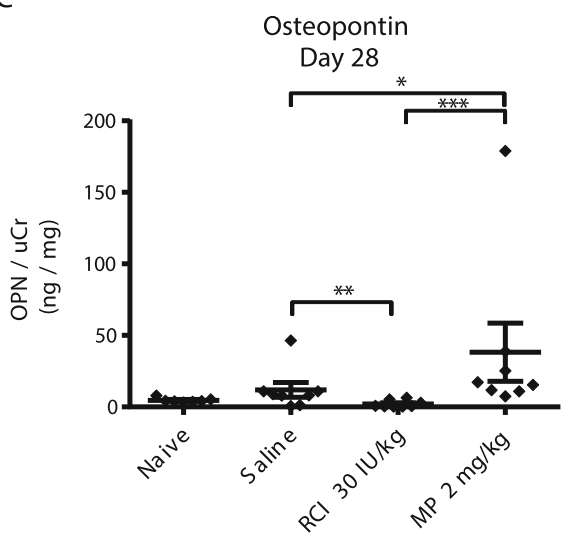

B

KIM-1

Day 56

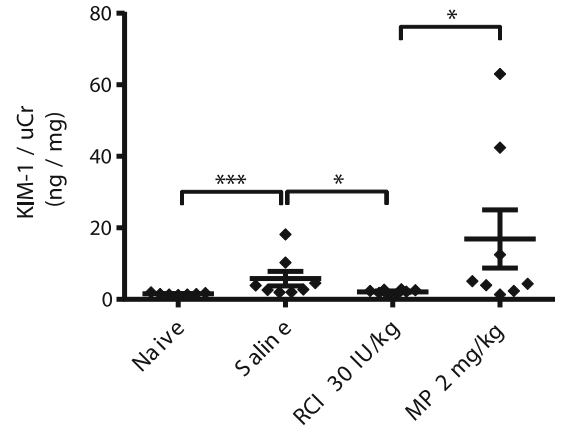

D

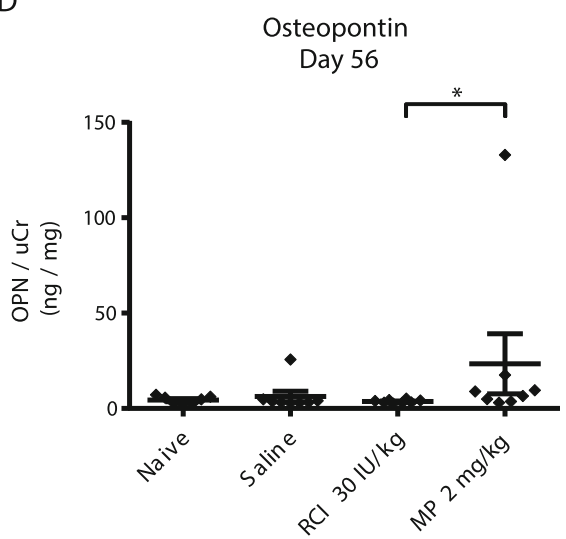

Fig. 3 Quantification of Urine OPN and KIM-1 Levels in the 8-week PAN Model. a, b KIM-1 levels normalized to creatinine. c, d OPN levels normalized to creatinine. For all panels, the Mann-Whitney test was used to determine statistical significance, and all values are mean \pm standard error of the mean. ${ }^{*} p<0.05 ;{ }^{* *} p<0.01 ;{ }^{* *} p<0.001$. Abbreviations: KIM-1, kidney injury molecule-1; MP, methylprednisolone; OPN, osteopontin; PAN, puromycin aminonucleoside; $\mathrm{RCl}$, repository corticotropin injection; $\mathrm{uCr}$, urine creatinine

significantly higher at day 42 in the 12 -week model $(p<$ 0.01 ) (Fig. 4e). RCI $30 \mathrm{IU} / \mathrm{kg}$ significantly reduced OPN levels $(p<0.01)$ at day 28 in the 8 -week model, whereas MP significantly increased OPN levels compared with saline $(p<0.05)$. Treatment with RCI significantly lowered OPN concentrations at day 42 for RCI 10, 30, and $60 \mathrm{IU} /$ $\mathrm{kg}(p<0.05)$ (Fig. 4e) and day 56 for RCI $60 \mathrm{IU} / \mathrm{kg}(p<$ 0.01 ) (Fig. 4f) compared with saline in the 12-week model.

In the 8-week model, RCI treatment protected against loss of PDPN expression, an indication of podocyte injury. PAN-induced renal injury decreased mean PDPN glomerular area staining by approximately $70 \%$ compared with naive (Fig. 5a, b). RCI $30 \mathrm{IU} / \mathrm{kg}$ treatment significantly increased PDPN glomerular expression compared with saline $(p<0.05)$, whereas MP treatment was unable to prevent PDPN loss, with lower mean area staining than saline (Fig. 5a, b). As a proportion of total glomerular area, glomerular PDPN staining was significantly negatively correlated to log-transformed 24-h urine protein (Pearson's $r=-0.77, p<0.0001$ ) (Fig. 6).
PDPN staining was evaluated in the 12-week model as well, but after the last PAN injection, PAN-injured animals showed normal expression of most podocyte markers comparable with naive controls after 7 weeks of recovery (data not shown).

\section{Effects of $\mathrm{RCI}$ on podocyte morphology in PAN-FSGS models}

RCI treatment led to long-term benefits in podocyte morphology as demonstrated by EM analysis in the 12week model. RCI treatment significantly decreased the prevalence of podocyte effacement (Fig. 7a-c) and total glomerular injury score in the $60 \mathrm{IU} / \mathrm{kg}$ dose group $(p<$ 0.05) compared to saline (Fig. 7d).

\section{Effects of $\mathrm{RCl}$ on fibrosis markers in PAN-FSGS models}

Quantitative image analysis of glomerular staining for epithelial-mesenchymal transition (EMT), a cellular mechanism of fibrosis [33], was also assessed in the kidney cortex in both models. Glomerular IHC staining of 


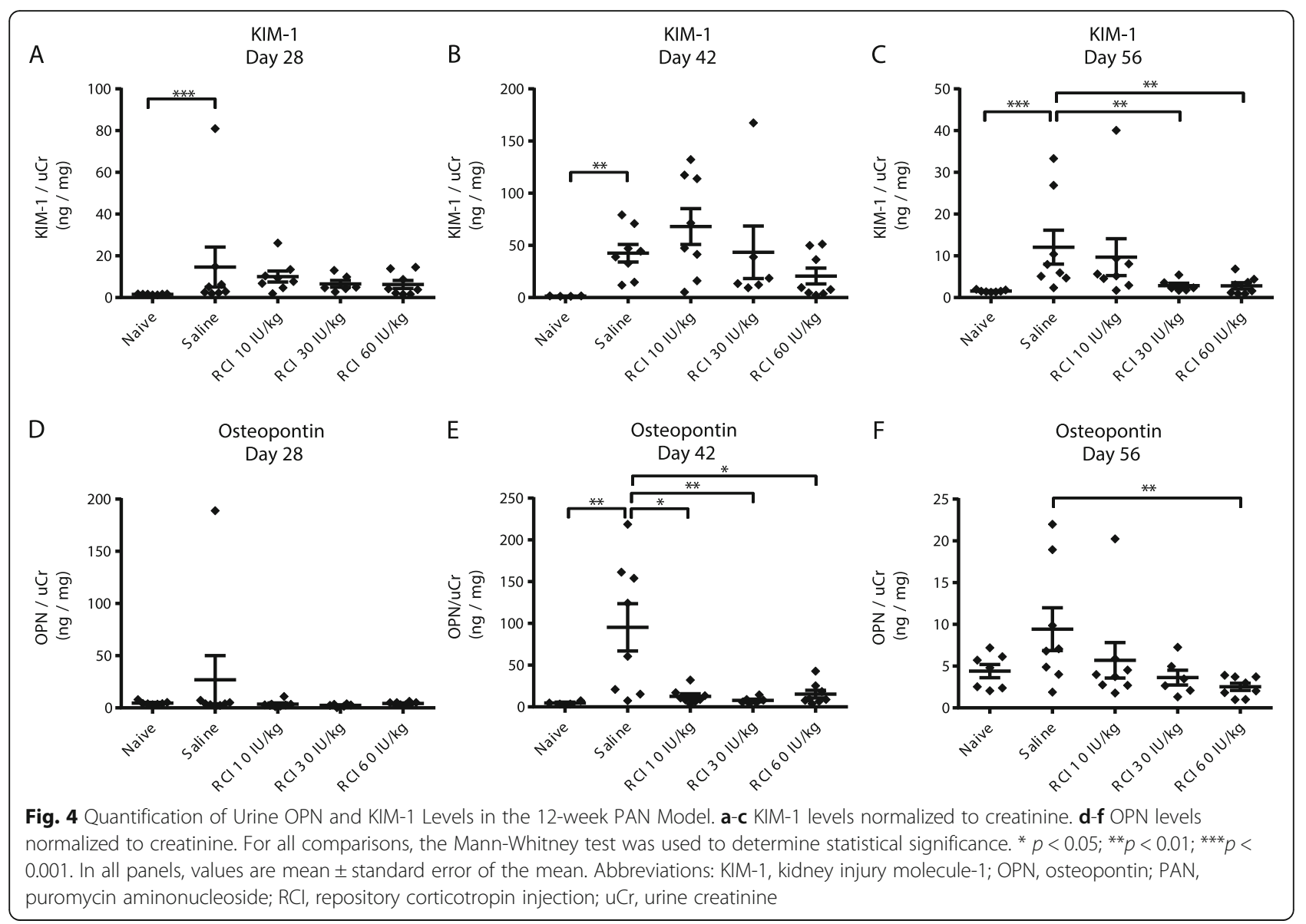

the EMT marker desmin was significantly elevated in PAN-treated animals $(p<0.0001)$ in both models (Fig. 8a, b). In the 12-week model, glomerular desmin staining was significantly lower for RCI $30 \mathrm{IU} / \mathrm{kg}(p<0.05)$ and $60 \mathrm{IU} / \mathrm{kg}(p<0.001)$ compared with saline (Fig. 8b). Sirius Red/Fast Green staining for collagen deposition, a marker for fibrosis, was significantly decreased with RCI $30 \mathrm{IU} / \mathrm{kg}$ versus saline $(p<0.05)$ in the 8 -week model (Fig. 8c) and for RCI $60 \mathrm{IU} / \mathrm{kg}$ versus saline $(p<0.01)$ in the 12-week model (Fig. 8d).

\section{Discussion}

Overall, RCI improved glomerular function and pathology after repeated PAN injury in Sprague-Dawley rats. RCI reduced proteinuria, improved EM measures of podocyte morphology, decreased urinary biomarkers of renal damage, and increased IHC markers of podocyte function. RCI also reduced histopathology scores of fibrosis, interstitial inflammation, tubular injury, and glomerular changes after injury. By comparison, MP treatment alone was not beneficial for any of the relevant endpoints in these models. The early benefit in proteinuria reduction by MP could not be maintained throughout the study, and overall renal injury in this group was no better than in saline controls, often with higher mean severity scores than no treatment.

Importantly, the improvements observed with RCI occur after podocyte damage in a rodent model of FSGS. Rather than simply preventing symptoms of FSGS, RCI was shown to reverse damage to podocytes, with improved podocyte structure and function even after repeated renal injury in the 12 -week model. These preclinical models demonstrate the therapeutic benefit of RCI in treatment of ongoing FSGS. The authors are not aware of another FSGS drug shown to improve damage to podocytes after repeated injury.

Results from this study are similar to published data showing statistically significant reductions in proteinuria with RCI in patients with FSGS [34, 35]. In addition, decreases in levels of KIM-1 (a tubular injury marker) and OPN (a glycoprotein associated with podocyte damage) with RCI treatment in this study are consistent with the previous characterization of KIM-1 and OPN as biomarkers of glomerular disease [36-38] and of acute kidney injury [39]. Although the increase in KIM-1 with MP compared with saline on days 28 and 56 was unexpected, 
A
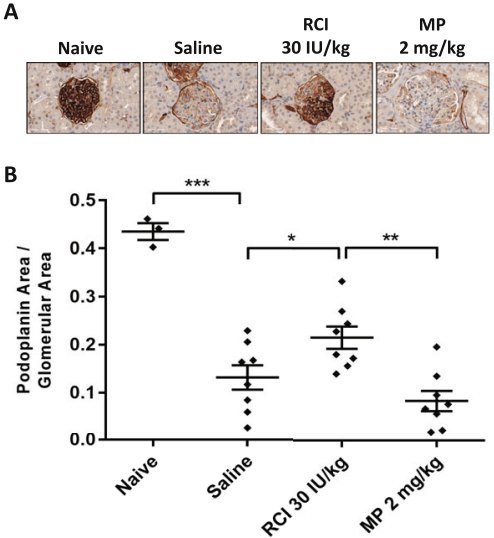

Fig. 5 Podoplanin Expression in the 8-Week PAN Model. a Podoplanin expression via $\mathrm{IHC}$ staining. $\mathbf{b} \mathrm{IHC}$ analysis of podoplanin levels presented as proportion of glomerular staining in kidney sections at day $\left.56{ }^{*} p<0.05 ;{ }^{* *} p<0.001 ;{ }^{* * *} p<0.0001\right)$. For all comparisons, unpaired t tests were used to determine statistical significance. Values are mean \pm standard error of the mean. Abbreviations: IHC, immunohistochemistry; MP, methylprednisolone; PAN, puromycin aminonucleoside; $\mathrm{RCl}$, repository corticotropin injection

the variance for KIM-1 in the MP groups was generally high, and the literature suggests that KIM-1 may have an unpredictable response to glucocorticoids. A patient with tubulointerstitial nephritis and uveitis showed fluctuations in KIM-1 after glucocorticoid treatment [40].

These results indicate that in an extended non-immune-mediated model of FSGS, steroid-mediated adverse effects from chronic treatment may outweigh early efficacy seen in FSGS models of shorter duration [41]. Although MP did not show sustained effect on proteinuria in this model, a randomized trial in patients with membranous nephropathy showed similar efficacy for RCI and MP on proteinuria remission [42]. The discrepancy in study results could be explained by differences across species or in study protocol; patients receiving MP in the membranous nephropathy study alternated the drug with cyclophosphamide or chlorambucil.

The increased PDPN expression demonstrated in this study may be a benefit of MCR-mediated RhoA activation, explored in previous studies [15-18]. The literature [43] and this study suggest that melanocortin receptor activation can improve renal disease with or without endogenous steroid production, with observed direct podocyte effects beyond general immunosuppression. The improvements in proteinuria and glomerular morphology observed in this study could be mediated specifically by RCIdependent activation of MC1R. In a rodent model of membranous nephropathy (passive Heymann nephritis),

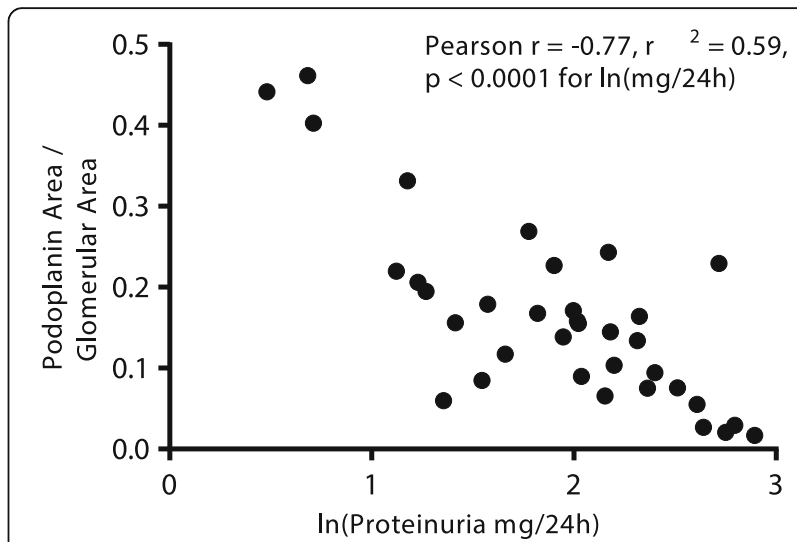

Fig. 6 Urine Protein Concentration and Podoplanin Staining per Glomerular Area Correlation in the 8-Week PAN Model. There is a significant negative correlation between glomerular podoplanin staining, as a proportion of total glomerular area, and logtransformed 24-h urine protein. The relationship between the two variables was quantified using the Pearson correlation coefficient and statistical significance was determined by $t$ test. Abbreviations: In, natural log; PAN, puromycin aminonucleoside

an MC1R agonist improved proteinuria and glomerular morphology [19]. However, in a rodent model of FSGS (adriamycin), MC1R agonists did not reduce albuminuria (a type of proteinuria) [20]. Differences in the animal models may account for these discrepancies. Further studies are warranted to identify specific RCI-dependent MCR activity in FSGS.

\section{Limitations}

Although a steroid treatment condition was included in the 8-week PAN group to model traditional steroid treatment, the 12-week PAN model did not include an MP group for comparison and was designed as a doseresponse study for RCI. The two studies also showed slight differences in baseline age and weight. Kidney function was evaluated by proteinuria; additional measures such as blood urea nitrogen, serum creatinine, and urinary albumin were not included.

\section{Conclusions}

In the current study, RCI treatment was effective in reducing PAN-induced renal damage and podocyte injury, which were not observed by treatment with MP. These results provide further evidence for a unique mechanism of MCR agonism in the treatment of FSGS, which was superior to steroid treatment in this experimental model. 


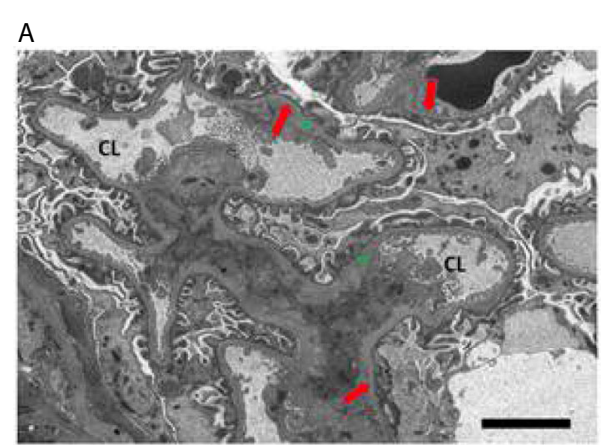

B

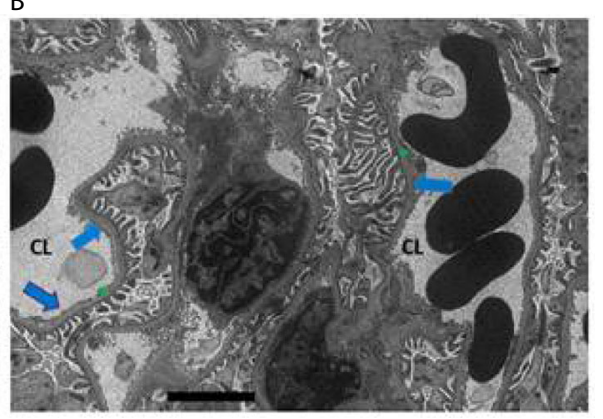

C

D
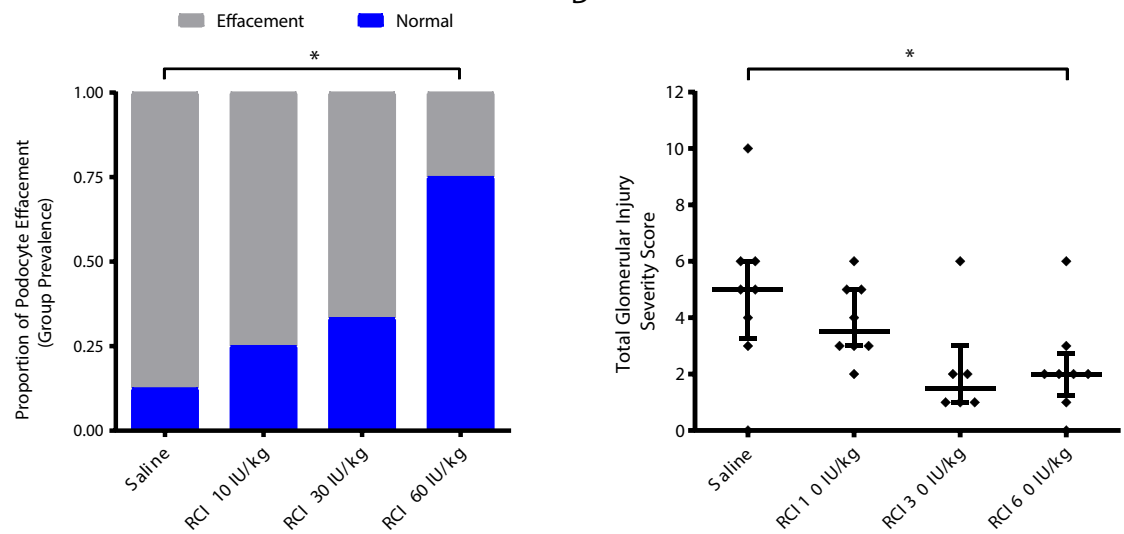

Fig. 7 Podocyte and Glomerular Assessment in the 12-week PAN Model. a Saline EM image. Red arrows= podocyte effacement. b RCl $60 \mathrm{IU} / \mathrm{kg}$ EM image. Blue arrows = normal podocyte foot process structure. $\mathbf{a}, \mathbf{b}$ Green ${ }^{*}=$ capillary basement membrane; $\mathrm{CL}=$ capillary lumen; scale bar = $4 \mu \mathrm{M}$. c Group prevalence of podocyte effacement by EM analysis. ${ }^{*} p<0.05$, Fisher's exact test for group differences compared with saline, $2-$ tailed. $\mathbf{d}$ Total glomerular injury score. ${ }^{*} p<0.05$, Kruskal-Wallis nonparametric ANOVA, Dunn's post hoc test, comparing the RCI treatment groups with saline. Values are mean \pm standard error of the mean. For all panels, naive samples are not shown because of low sample size. Abbreviations: ANOVA, analysis of variance; EM, electron microscopy; PAN, puromycin aminonucleoside; RCl, repository corticotropin injection 


\section{8-Week PAN Model}
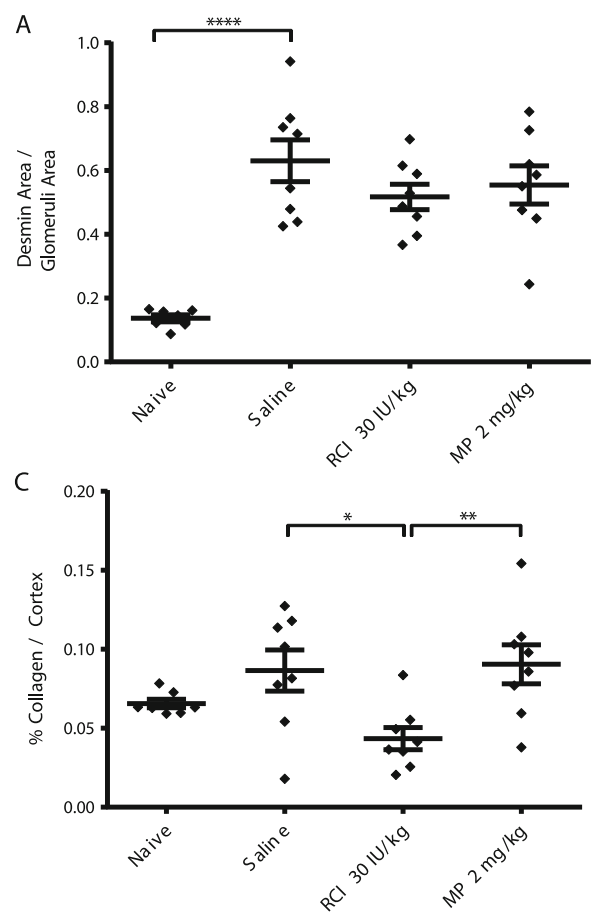

12-Week PAN Model

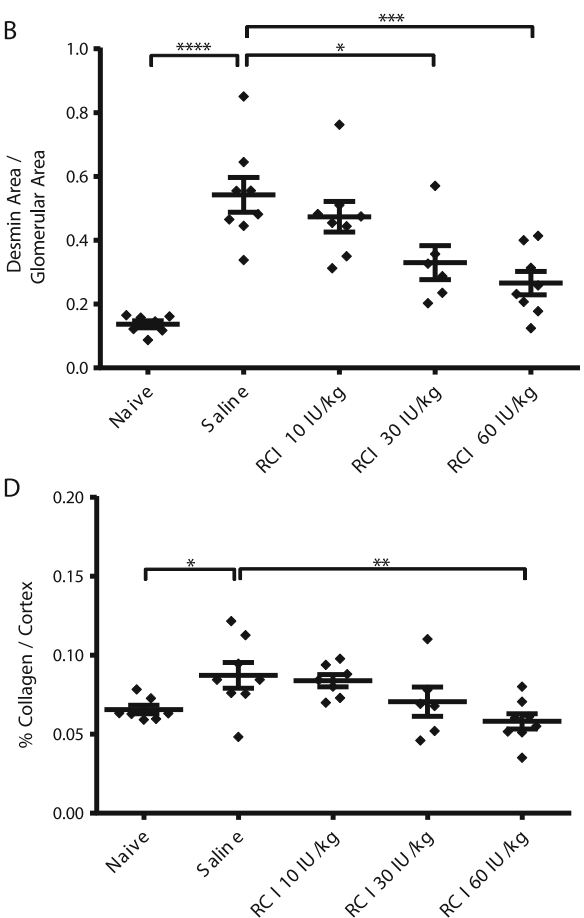

Fig. 8 Fibrosis Marker Levels in the 8-week and 12-week PAN Models. a, b Image analysis of glomerular staining for desmin as a marker of epithelial-mesenchymal transition. $\mathbf{c}$, d Sirius Red/Fast Green (collagen) deposition staining as a marker of fibrosis. For all comparisons, unpaired $t$ tests were used to determine statistical significance. ${ }^{*} p<0.05 ;{ }^{* *} p<0.01 ;{ }^{* * *} p<0.001 ;{ }^{* * *} p<0.0001$. Values are mean \pm standard error of the mean. Abbreviations: MP, methylprednisolone; PAN, puromycin aminonucleoside; $\mathrm{RCl}$, repository corticotropin injection

\section{Abbreviations}

ANOVA: Analysis of variance; AUC: Area under the curve; ELISA: Enzymelinked immunosorbent assay; EM: Electron microscopy; EMT: Epithelialmesenchymal transition; FSGS: Focal segmental glomerulosclerosis; H\&E: Haematoxylin and eosin; iMN: Idiopathic membranous nephropathy; IHC: Immunohistochemistry; IV: Intravenous; KIM-1: Kidney injury molecule-1; MCR: Melanocortin receptor; MP: Methylprednisolone; MS: Multiple sclerosis; NF-KB: Nuclear factor-kappa B; NS: Nephrotic syndrome; OPN: Osteopontin; PAN: Puromycin aminonucleoside; PAS: Periodic acid-Schiff; PDPN: Podoplanin; RCI: Repository corticotropin injection; ROI: Region of interest; uCr: Urine creatinine

\section{Acknowledgments}

Editorial support and scientific consulting for this manuscript were provided by MedLogix Communications, LLC, Itasca, Illinois.

\section{Authors' contributions}

Study concept and design: KH, DW, and RMF. Performed and analyzed experiments: KH, EW, and CB. Manuscript draft and edits: $\mathrm{KH}, \mathrm{EW}, \mathrm{CB}, \mathrm{DW}$, and RMF. All authors approved the final version of the manuscript.

\section{Funding}

This study was funded by Mallinckrodt Pharmaceuticals, who had a role in the design of the study and collection, analysis, and interpretation of data as well as in writing the manuscript.

\section{Availability of data and materials}

The datasets used and/or analyzed during the current study are available from the corresponding author on reasonable request.

\section{Ethics approval and consent to participate}

All study procedures, including for animal use welfare, were in accordance with the National Institute of Health's Guide for the Care and Use of
Laboratory Animals. The study protocol was reviewed and approved by the Institutional Animal Care and Use Committee of Mallinckrodt Pharmaceuticals.

\section{Consent for publication}

Not applicable.

Competing interests

All of the authors are employees of Mallinckrodt Pharmaceuticals.

Received: 22 November 2019 Accepted: 2 June 2020

Published online: 16 July 2020

\section{References}

1. Malaga-Dieguez L, Bouhassira D, Gipson D, Trachtman H. Novel therapies for FSGS: preclinical and clinical studies. Adv Chronic Kidney Dis. 2015;22(2): e1-6.

2. Peev V, Hahm E, Reiser J. Unwinding focal segmental glomerulosclerosis. F1000Res. 2017;6:466.

3. Beaudreuil S, Lorenzo HK, Elias M, Nnang Obada E, Charpentier B, Durrbach A. Optimal management of primary focal segmental glomerulosclerosis in adults. Int J Nephrol Renovasc Dis. 2017;10:97-107.

4. Haas M, Meehan SM, Karrison TG, Spargo BH. Changing etiologies of unexplained adult nephrotic syndrome: a comparison of renal biopsy findings from 1976-1979 and 1995-1997. Am J Kidney Dis. 1997;30(5):621-31.

5. Sim JJ, Bhandari SK, Batech M, Hever A, Harrison TN, Shu YH, et al. Endstage renal disease and mortality outcomes across different glomerulonephropathies in a large diverse US population. Mayo Clin Proc. 2018:93(2):167-78

6. Kidney Disease: Improving Global Outcomes (KDIGO) Glomerulonephritis Work Group. KDIGO clinical practice guideline for glomerulonephritis. Kidney Int Suppl. 2012;2(2):139-274. 
7. de Haij S, Daha MR, van Kooten C. Mechanism of steroid action in renal epithelial cells. Kidney Int. 2004;65(5):1577-88.

8. Hogan J, Radhakrishnan J. The treatment of idiopathic focal segmental glomerulosclerosis in adults. Adv Chronic Kidney Dis. 2014;21(5):434-41.

9. Tumlin JA, Campbell KN. Proteinuria in nephrotic syndrome: mechanistic and clinical considerations in optimizing management. Am J Nephrol. 2018; 47(Suppl 1):1-2.

10. Campbell KN, Tumlin JA. Protecting podocytes: a key target for therapy of focal segmental glomerulosclerosis. Am J Nephrol. 2018;47(Suppl 1):14-29.

11. Acthar Gel. Package insert. Mallinckrodt ARD LLC; 2019.

12. Tumlin J, Galphin C, Santos R, Rovin B. Safety and efficacy of combination ACTHar gel and tacrolimus in treatment-resistant focal segmental glomerulosclerosis and membranous glomerulopathy. Kidney Int Rep. 2017; 2(5):924-32.

13. Bomback AS, Tumlin JA, Baranski J, Bourdeau JE, Besarab A, Appel AS, et al. Treatment of nephrotic syndrome with adrenocorticotropic hormone (ACTH) gel. Drug Des Devel Ther. 2011;5:147-53.

14. Bomback AS, Fervenza FC. Membranous nephropathy: approaches to treatment. Am J Nephrol. 2018;47(Suppl 1):30-42.

15. Chida D, Nakagawa S, Nagai S, Sagara H, Katsumata H, Imaki T, et al. Melanocortin 2 receptor is required for adrenal gland development, steroidogenesis, and neonatal gluconeogenesis. Proc Natl Acad Sci U S A. 2007;104(46):18205-10.

16. Herman JP, McKlveen JM, Ghosal S, Kopp B, Wulsin A, Makinson R, et al. Regulation of the hypothalamic-pituitary-adrenocortical stress response. Compr Physiol. 2016;6(2):603-21.

17. Catania A, Gatti S, Colombo G, Lipton JM. Targeting melanocortin receptors as a novel strategy to control inflammation. Pharmacol Rev. 2004;56(1):1-29.

18. Lisak RP, Benjamins JA. Melanocortins, melanocortin receptors and multiple sclerosis. Brain Sci. 2017;7(8):E104.

19. Lindskog A, Ebefors K, Johansson ME, Stefánsson B, Granqvist A, Arnadottir $M$, et al. Melanocortin 1 receptor agonists reduce proteinuria. J Am Soc Nephrol. 2010;21(8):1290-8

20. Lindskog Jonsson A, Granqvist A, Elvin J, Johansson ME, Haraldsson B, Nyström J. Effects of melanocortin 1 receptor agonists in experimental nephropathies. PLoS One. 2014;9(1):e87816.

21. Elvin J, Buvall L, Lindskog Jonsson A, Granqvist A, Lassén E, Bergwall L, et al. Melanocortin 1 receptor agonist protects podocytes through catalase and RhoA activation. Am J Physiol Renal Physiol. 2016;310(9):F846-F56.

22. Bergwall L, Wallentin H, Elvin J, Liu P, Boi R, Sihlbom C, et al. Amplification of the melanocortin-1 receptor in nephrotic syndrome identifies a target for podocyte cytoskeleton stabilization. Sci Rep. 2018;8(1):15731.

23. Lal R, Bell S, Challenger R, Hammock V, Nyberg M, Decker D, et al. Pharmacodynamics and tolerability of repository corticotropin injection in healthy human subjects: a comparison with intravenous methylprednisolone. J Clin Pharmacol. 2016;56(2):195-202.

24. Filippone EJ, Dopson SJ, Rivers DM, Monk RD, Udani SM, Jafari G, et al. Adrenocorticotropic hormone analog use for podocytopathies. Int Med Case Rep J. 2016;9:125-33.

25. Gong R. The renaissance of corticotropin therapy in proteinuric nephropathies. Nat Rev Nephrol. 2011;8(2):122-8.

26. Hogan J, Bomback AS, Mehta K, Canetta PA, Rao MK, Appel GB, et al. Treatment of idiopathic FSGS with adrenocorticotropic hormone gel. Clin J Am Soc Nephrol. 2013;8(12):2072-81.

27. Allinovi M, De Chiara L, Angelotti ML, Becherucci F, Romagnani P. Antifibrotic treatments: a review of clinical evidence. Matrix Biol. 2018;68-69: 333-54.

28. Fogo AB. Animal models of FSGS: lessons for pathogenesis and treatment. Semin Nephrol. 2003;23(2):161-71.

29. Suzuki K, Fukusumi Y, Yamazaki M, Kaneko H, Tsuruga K, Tanaka H, et al. Alteration in the podoplanin-ezrin-cytoskeleton linkage is an important initiation event of the podocyte injury in puromycin aminonucleoside nephropathy, a mimic of minimal change nephrotic syndrome. Cell Tissue Res. 2015;362(1):201-13.

30. Erdely A, Freshour G, Smith C, Engels K, Olson JL, Baylis C. Protection against puromycin aminonucleoside-induced chronic renal disease in the WistarFurth rat. Am J Physiol Renal Physiol. 2004;287(1):F81-9.

31. Charan J, Kantharia ND. How to calculate sample size in animal studies? J Pharmacol Pharmacother. 2013;4(4):303-6.

32. Korbet SM. Treatment of primary FSGS in adults. J Am Soc Nephrol. 2012; 23(11):1769-76.
33. Kalluri R, Weinberg RA. The basics of epithelial-mesenchymal transition. J Clin Invest. 2009;119(6):1420-8.

34. Madan A, Mijovic-Das S, Stankovic A, Teehan G, Milward AS, Khastgir A. Acthar gel in the treatment of nephrotic syndrome: a multicenter retrospective case series. BMC Nephrol. 2016;17:37.

35. Alhamad T, Manllo Dieck J, Younus U, Matar D, Alasfar S, Vujjini V, et al. ACTH Gel in resistant focal segmental glomerulosclerosis after kidney transplantation. Transplantation. 2019;103(1):202-9.

36. Zhang Q, Jiang C, Tang T, Wang H, Xia Y, Shao Q, et al. Clinical significance of urinary biomarkers in patients with primary focal segmental glomerulosclerosis. Am J Med Sci. 2018;355(4):314-21.

37. Lorenzen J, Shah R, Biser A, Staicu SA, Niranjan T, Garcia AM, et al. The role of osteopontin in the development of albuminuria. J Am Soc Nephrol. 2008; 19(5):884-90.

38. Schordan S, Schordan E, Endlich K, Endlich N. AlphaV-integrins mediate the mechanoprotective action of osteopontin in podocytes. Am J Physiol Renal Physiol. 2011;300(1):F119-32.

39. Vaidya VS, Ferguson MA, Bonventre JV. Biomarkers of acute kidney injury. Annu Rev Pharmacol Toxicol. 2008;48:463-93.

40. Sugiyama M. Measurement of urinary biomarkers in a case of tubulointerstitial nephritis and uveitis syndrome during glucocorticoid treatment. CEN Case Rep. 2018:7(2):221-4.

41. Zhang J, Pippin JW, Krofft RD, Naito S, Liu ZH, Shankland SJ. Podocyte repopulation by renal progenitor cells following glucocorticoids treatment in experimental FSGS. Am J Physiol Renal Physiol. 2013;304(11):F1375-F89.

42. Ponticelli C, Passerini P, Salvadori M, Manno C, Viola BF, Pasquali S, et al. A randomized pilot trial comparing methylprednisolone plus a cytotoxic agent versus synthetic adrenocorticotropic hormone in idiopathic membranous nephropathy. Am J Kidney Dis. 2006;47(2):233-40.

43. Qiao Y, Berg AL, Wang P, Ge Y, Quan S, Zhou S, et al. MC1R is dispensable for the proteinuria reducing and glomerular protective effect of melanocortin therapy. Sci Rep. 2016;6:27589.

\section{Publisher's Note}

Springer Nature remains neutral with regard to jurisdictional claims in published maps and institutional affiliations.

Ready to submit your research? Choose BMC and benefit from:

- fast, convenient online submission

- thorough peer review by experienced researchers in your field

- rapid publication on acceptance

- support for research data, including large and complex data types

- gold Open Access which fosters wider collaboration and increased citations

- maximum visibility for your research: over $100 \mathrm{M}$ website views per year

At BMC, research is always in progress.

Learn more biomedcentral.com/submissions 